\title{
Especies del género Bacillus: morfología macroscópica y microscópica
}

\author{
María Elena Realpe ${ }^{1}$, Carlos Arturo Hernández ${ }^{2}$, Clara Inés Agudelo ${ }^{1}$ \\ ${ }^{1}$ Grupo Microbiología, Instituto Nacional de Salud, Bogotá, D.C., Colombia \\ 2 División de Biblioteca y Publicaciones, Instituto Nacional de Salud, Bogotá, D.C., Colombia \\ Entre octubre de 2001 y enero de 2002, el Grupo de Microbiología del Instituto Nacional de \\ Salud procesó 705 sobres remitidos por sospechas de contener esporas de ántrax. Presentamos \\ las fotografías de los cultivos de las diferentes especies de Bacillus spp. y de las láminas \\ hechas a partir de los cultivos para que sirvan como referencia para la correcta identificación \\ macro y microscópica del agente hallado en las muestras enviadas para confirmación.
}

Palabras clave: Bacillus spp, Bacillus anthracis, Bacillus cereus, Bacillus thuringiensis, Bacillus sphaericus, Bacillus subtilis, morfología macroscópica, morfología microscópica.

\section{Bacillus species: macroscopic and microscopic morphology}

Between October 2001 and January 2002, the Microbiology Group of the Instituto Nacional de Salud processed 705 envelopes under suspicion of harboring anthrax spores. We present photograhs of cultures and slides prepared from them of Bacillus species to be used as reference material for the accurate macroscopic and microscopic identification of the agent found in samples.

Key words: Bacillus spp, Bacillus anthacis, Bacillus cereus, Bacillus thuringiensis, Bacillus sphaericus, Bacillus subtilis, macroscopic morphology, microscopic morphology

La confirmación del primer caso de ántrax en Estados Unidos el pasado 4 de octubre como resultado de un acto terrorista trajo consigo la necesidad de confirmar por cultivo o por microscopía la presencia de Bacillus anthracis en las muestras obtenidas de sobres sospechosos de contener las esporas del agente. Hasta diciembre de 2001, en Estados Unidos se habían diagnosticado 11 casos pulmonares y 11 cutáneos según criterios clínicos y de laboratorio; todos los casos estaban relacionados con la manipulación de sobres de correo contaminados con las esporas del bacilo.

El Instituto Nacional de Salud organizó un sistema de información disponible en el portal del INS (www.ins.gov.co) para cubrir las necesidades del público en general. Simultáneamente, el Grupo de Microbiología, con estrecha colaboración de

\section{Correspondencia:}

Clara Inés Agudelo, Grupo de Microbiología, Instituto Nacional de Salud. Avenida Calle 26 \#51-60 tel 2207700 (ext 445).cagudelov@hemagogus.ins.gov.co

Recibido: 28/02/02; aceptado: 23/05/02
13 Laboratorios Departamentales de Salud Pública (LDSP), inició el estudio de sobres provenientes de diversos lugares del país sospechosos de contener esporas de ántrax; desde octubre de 2001 hasta enero de 2002, se procesaron 705 sobres, 209 en el Grupo de Microbiología y 496 en los LDSP; todos los resultados fueron negativos para $B$. anthracis.

El material obtenido de los sobres en estudio se cultivó en agar sangre de cordero al $5 \%$ y después se incubó a $35^{\circ} \mathrm{C}$ en aerobiosis durante 24 horas con el fin de obtener colonias de Bacillus $s p$. A partir de las colonias, se realizó la coloración de Gram a las 24 y 48 horas de incubación con el fin de observar la morfología microscópica (1.000X) de la especie de bacilo presente en el cultivo.

A continuación se presentan las imágenes macroscópicas de las colonias y las microscópicas de Bacillus anthracis, Bacillus cereus, Bacillus sphaericus y Bacillus subtilis para que sirvan de consulta para la confirmación correcta del agente hallado en las muestras remitidas para estudio. 
Bacillus anthracis
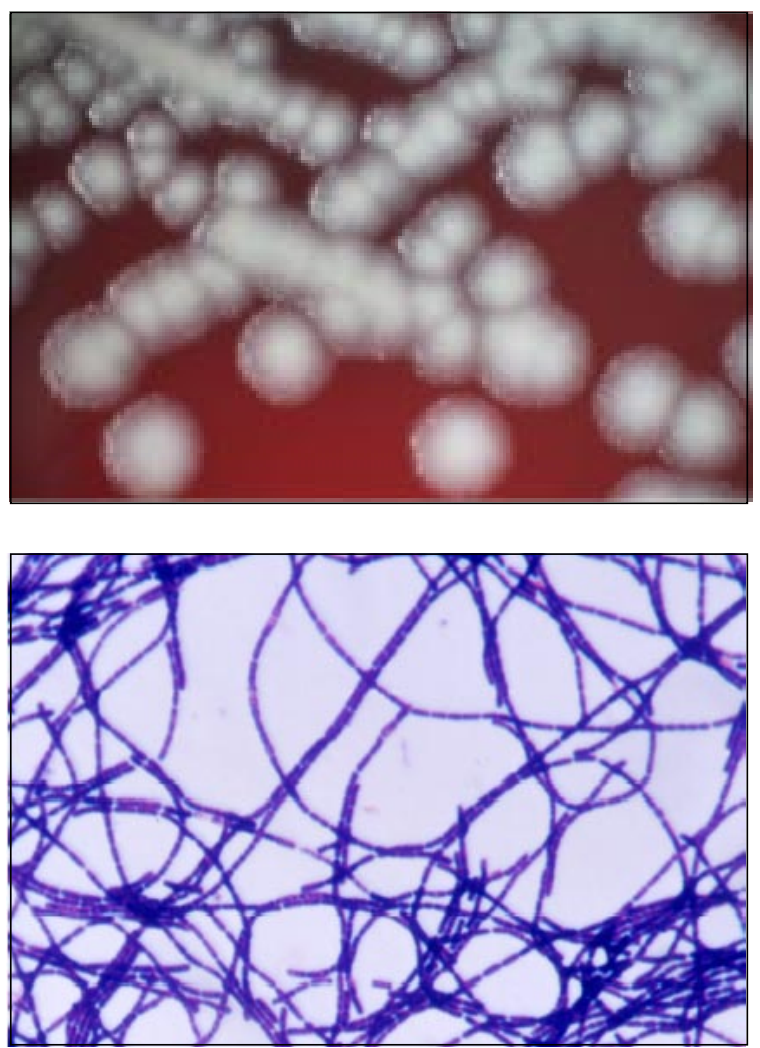

Gram 24 horas

\section{Bacillus cereus}

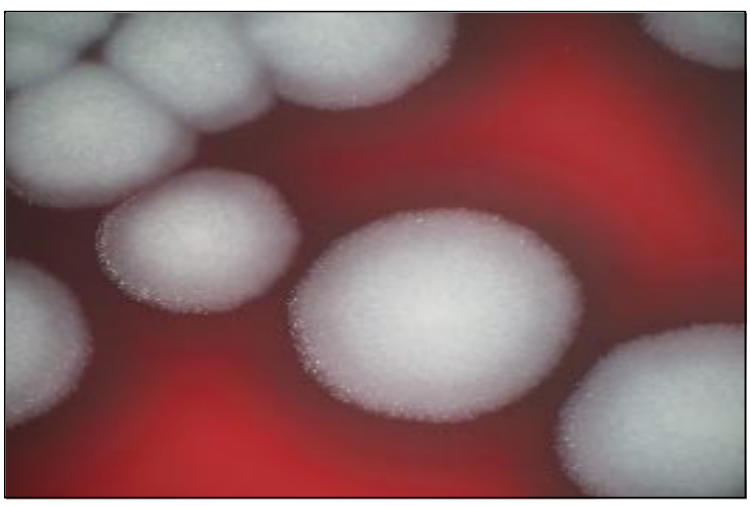

Colonias de 3-8 mm de diámetro, beta hemolíticas con hemólisis completa, de color gris a verde, aspecto de vidrio esmerilado y márgenes onduladas. Las colonias se extienden por el medio.
Colonias no hemolíticas de 4 a $5 \mathrm{~mm}$ de diámetro, con apariencia de vidrio esmerilado y bordes irregulares. Las colonias se extienden sobre el medio.

Gram 24 horas: bacilos Gram positivos, de 1 a 1,3 $\mathrm{mm}$ de diámetro por 3 a $10 \mathrm{~mm}$ de largo, con bordes cuadrados 0 cóncavos que forman cadenas largas con la apariencia de vara de bambú.

Gram 48 horas: bacilos Gram positivos con esporas de forma elipsoidal en posición subterminal que no deforman el bacilo.

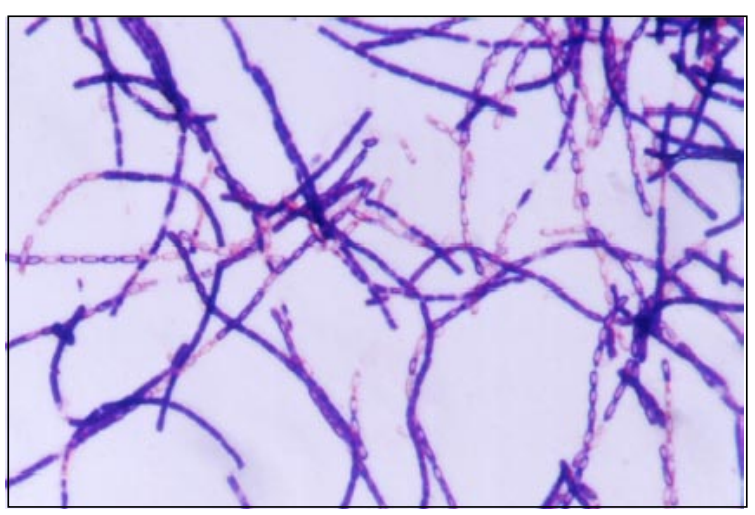

Gram 48 horas

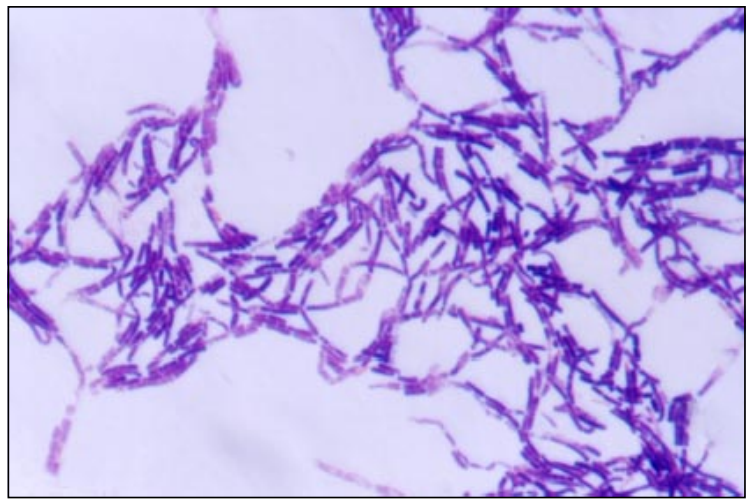

Gram 24 horas: bacilos Gram positivos de 1,3 $\mathrm{mm}$ de diámetro por 3 a $5 \mathrm{~mm}$ de largo con bordes redondeados, que forman cadenas cortas. Se observan esporas cilíndricas subterminales. 


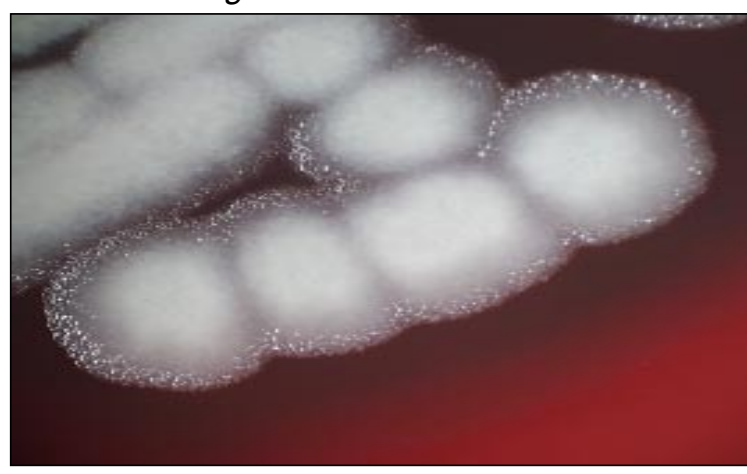

Colonias de 3 a $8 \mathrm{~mm}$ de diámetro betahemolíticas con hemólisis completa, de color de gris a verde, con aspecto de vidrio esmerilado y bordes regulares, que forman agrupaciones en cadenas que se pueden observar macroscópicamente.

\section{Bacillus sphaericus}

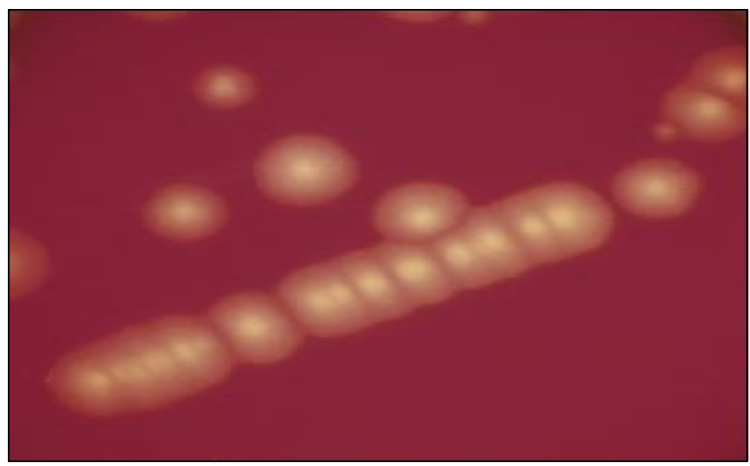

Colonias de 2 a $4 \mathrm{~mm}$ de diámetro, beta hemolíticas con hemólisis completa, de aspecto liso, color blanco crema translúcido y bordes regulares.

\section{Bacillus subtilis}

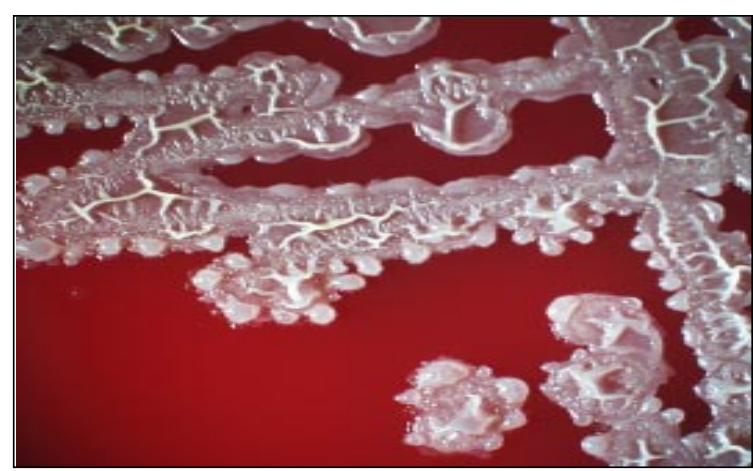

Colonias, de 2 a $4 \mathrm{~mm}$ de diámetro, beta hemolíticas con hemólisis completa, que pueden ser de aspecto liso, mucoide o rugoso; los bordes pueden ser ondulados o extendidos en el medio y ocasionalmente dan la apariencia de cultivos mixtos.

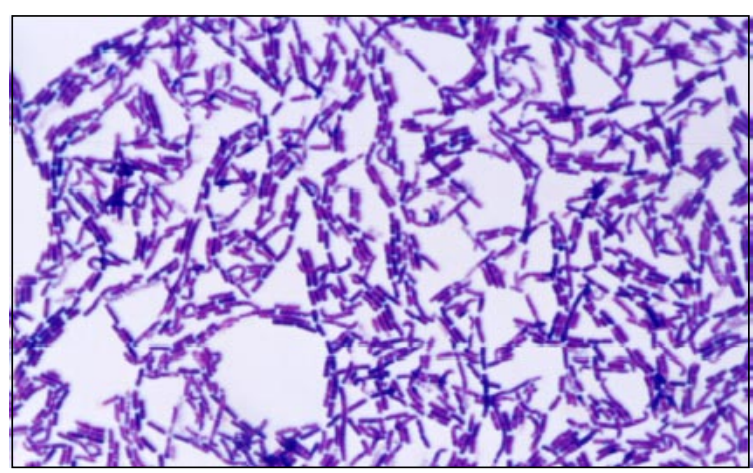

Gram 24 horas: bacilos Gram positivos, de 1,2 $\mathrm{mm}$ de diámetro por 3 a $5 \mathrm{~mm}$ de largo, con borde redondeados y que forman cadenas cortas. Se observan esporas elipsoidales y centrales que no deforman el bacilo.

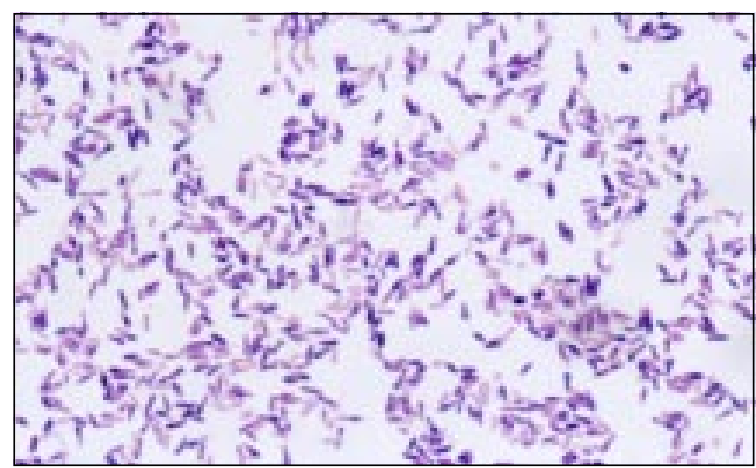

Gram 24 horas: bacilos Gram positivos de $1 \mathrm{~mm}$ de diámetro por 1,5 a $5 \mathrm{~mm}$ de largo, con bordes redondeados. Se observan esporas esféricas, terminales, que deforman el bacilo y le dan aspecto de raqueta.

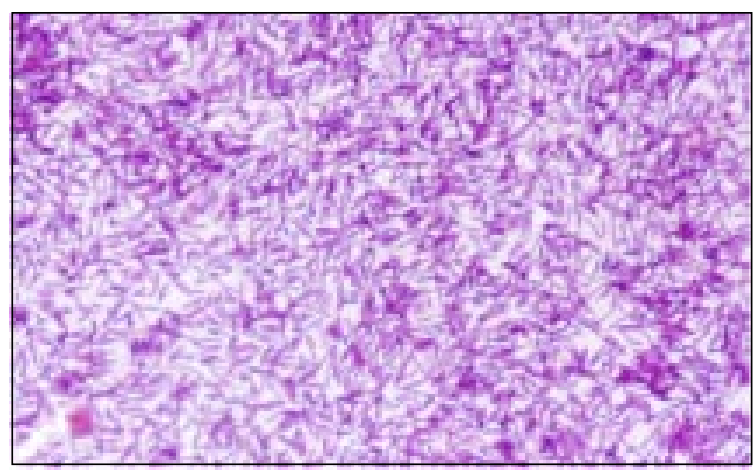

Gram 24 horas: bacilos Gram positivos de $0,8 \mathrm{~mm}$ de diámetro por 2 a $3 \mathrm{~mm}$ de largo con bordes redondeados. Presentan esporas esféricas y centrales que no deforman el bacilo. 


\section{Agradecimientos}

A la doctora Elizabeth Castañeda del Grupo de Microbiología por su valiosa colaboración y al Grupo de Entomología por la colaboración en la toma de las fotografías.

\section{Lecturas recomendadas}

1. Logan NA, Turnbull CBP. Bacillus and recently derived genera. En: Murray PR, Baron EJ, Pfaller MA, Tenover $\mathrm{FC}$, Yolken $\mathrm{RH}$, editors. Manual of clinical microbiology. Seventh edition. Washington, D.C.: ASM Press; 1999. p. 357-69.

En este capítulo se encuentra una descripción completa del género Bacillus y las diferencias de sus especies tanto morfológicas como bioquímicas.

2. Koneman EW, Allen SD, Janda WM, Schreckenberger PC, Winn WC Jr, editores. Color atlas and textbook of diagnostic microbiology. Fifth edition. Philadelphia, PA.: Lippincott Willians and Wilkins; 1997 p.651-708.

En este capítulo se presenta una descripción completa del género Bacillus y las diferencias de sus especies tanto morfológicas como bioquímicas.

3. Young JAT, Collier RJ. Attacking anthrax. Sci Am 2002;286:36-45.
Revisión de la acción de la toxina de Bacillus anthracis y sus posibilidades de tratamiento por medio de mezclas de antitoxinas y antibióticos.

4. Dixon TC, Meselson M, Guillemin J, Hanna PC. Anthrax. N Eng J Med 1999;341:815-26.

Revisión del ántrax que incluye patogénesis, manifestaciones clínicas, diagnóstico, prevención y tratamiento.

5. Shafazand S, Doyle R, Ruoss S, Weunacker A, Raffin TA. Anthrax inhalational: epidemiology, diagnosis and management. Chest 1999;116:1369-76.

Revisión clínica del ántrax pulmonar.

6. Centers for Disease Control and Prevention. Basic laboratory protocolos for presuntive identification of Bacillus anthracis. April 18 of 2001. (www.cdc.gov)

Se describen las técnicas para el diagnóstico del ántrax $y$ las medidas de bioseguridad.

7. World Health Organization. Guidelines for the surveillance and control of anthrax in human and animals. Geneva: World Health Organization. Emerging and other communicable diseases, surveillance and control.

Tomado de página web de la WHO (www.who.int/emc). Se describen las técnicas para el diagnóstico del ántrax y las medidas de bioseguridad; además, describe la enfermedad y el sistema de vigilancia y notificación. 